\title{
Fluorodopa F 18
}

National Cancer Institute

\section{Source}

National Cancer Institute. Fluorodopa F18. NCI Thesaurus. Code C95766.

The amino acid analog fluorodopa (FDOPA) labeled with fluorine $\mathrm{F} 18$, a positron-emitting isotope, with potential tumor tracer property. Fluorine $F 18$ fluorodopa is able to cross the blood-brain barrier and is taken up by brain tumor cells. As uptake is higher in tumor cells, tumors may then be imaged using positron emission tomography (PET). Assessing tumor uptake of FDOPA may be beneficial for diagnosis, localization and in determining further treatment. 